\title{
Research Highlights A Role for Prefrontal Cortical NMDA Receptors in Murine Alcohol-Heightened Aggression
}

\author{
Mary M Torregrossa*,I \\ 'Department of Psychiatry, University of Pittsburgh, Pittsburgh, PA, USA
}

Neuropsychopharmacology (2018) 43, I199-1200; doi:I0.1038/npp.20 17.286; published online 10 January 2018

Our social lexicon includes numerous phrases to describe how individuals behave under the influence of alcohol. In particular, people often refer to 'sad drunks,' 'happy drunks,' and to 'angry drunks.' These descriptions illustrate that we intuitively recognize the large variation across the population in how individuals behave under the influence of alcohol. Moreover, the tendency for alcohol to elicit anger and aggression in some individuals is particularly problematic in our society, as up to $50 \%$ of violence-related injuries in the world involve the use of alcohol (Cherpitel et al, 2012). Therefore, increased understanding of the neurobiological underpinnings of why some individuals develop alcoholheightened aggression could lead to targeted behavioral or pharmacological approaches to drastically reduce violence in our society. Intriguingly, individual variation in the behavioral response to alcohol is not limited to humans. Indeed, mice, rats, and monkeys can all exhibit alcohol-heightened aggression, and, as with humans, this form of aggression is only observed in a small subset of the population (van Erp and Miczek, 1997; Miczek et al, 1998). Thus, it is possible to use animal models to determine what biological factors cause some individuals to become violent under the influence of alcohol, providing a means for developing preventative strategies.

In this issue of Neuropsychopharmacology, Newman et al (2018) took advantage of individual differences in the expression of alcohol-heightened aggression in mice to identify novel mechanisms underlying this behavioral vulnerability. The experiments focused on determining if alcohol-heightened aggressor mice (AHAs) differed from alcohol non-heightened aggressor mice (ANAs) in their expression of glutamatergic NMDA receptor subunits and their behavioral response to NMDAR inhibition. Alcohol has diverse effects on the central nervous system, however, inhibition of NMDAR signaling is thought to be involved in many of the behavioral manifestations of alcohol intoxication (Morisot and Ron, 2017). Therefore, differential

\footnotetext{
*Correspondence: Dr MM Torregrossa, Department of Psychiatry, University of Pittsburgh, Bridgeside Point II, Room 228, 450 Technology Drive, Pittsburgh, PA 15219, USA, Tel: 412624 5723, Fax: 412624 5280, E-mail: torregrossam@upmc.edu
}

sensitivity or function of NMDA receptors could explain individual variation in alcohol-heightened aggression. Specifically, NMDARs in the prefrontal cortex (PFC) are located on both GABAergic interneurons and pyramidal cells, and Newman and colleagues hypothesized that the balance of NMDAR-mediated inhibition $v s$ disinhibition of the PFC could regulate individual differences in the expression of aggressive behavior.

In order to test this hypothesis, the researchers first screened male mice for alcohol-heightened aggression. All mice were housed in breeding pairs so that males would show species-typical aggression toward an intruder mouse after removal of the female. Mice were also trained to drink $1 \mathrm{~g} / \mathrm{kg}$ of $6 \%$ alcohol $(\mathrm{EtOH})$ or an equivalent volume of water during brief, limited access periods. The propensity for alcohol-heightened aggression was then assessed across six sessions that alternated between a water access period or an alcohol access period immediately prior to introduction of the intruder mouse. Attack bite frequency and topography were analyzed within subject by comparing alcohol to water access sessions. Mice defined as AHAs exhibited a twofold increase in bite frequency after consuming alcohol, while ANA mice did not differ between alcohol and water sessions. Importantly, AHA and ANA mice did not differ in aggressive behavior in control conditions, indicating that AHAs were not more aggressive overall, but rather had a selective sensitivity to alcohol-heightened aggression. In addition, to increased bite frequency, AHAs also exhibited a change in bite topography, shifting from species-typical attacks to the posterior back to increased attacks on the anterior back. Finally, AHAs and ANAs did not differ in alcohol consumption or metabolism, suggesting that other biological factors underlie this individual difference.

Next, the researchers measured NMDAR subunit expression in several brain regions. AHAs and ANAs did not differ in the expression of GluN1, GluN2B, or GluN2A in any region examined. However, in the $\mathrm{MPFC}$, AHA mice showed significantly increased levels of GluN2D relative to ANAs. Therefore, PFC-specific GluN2D signaling may be involved in alcohol-heightened aggression, which is particularly interesting given that GluN2D receptors may be preferentially expressed 
on parvalbumin interneurons (Povysheva and Johnson, 2016). In order to further determine if NMDAR signaling differences account for the expression of alcohol-heightened aggression, the researchers determined if other NMDAR antagonists produced effects similar to alcohol in the two populations. They tested the effects of systemic administration of ketamine and memantine, two uncompetitive NMDAR channel blockers, on aggressive behavior in the presence or absence of alcohol exposure. In AHA mice, both ketamine and memantine when given alone at moderate doses, increased aggressive behavior, effectively mimicking the effects of alcohol. In ANA mice, no change in aggressive behavior was observed in response to moderate doses of ketamine or memantine, in accordance with the absence of alcohol-induced aggression in these mice. In contrast, when mice drank alcohol in addition to receiving ketamine or memantine, AHA mice showed no change or reduced aggression, while ANA mice showed increased aggression when alcohol and memantine were given together. Therefore, it appears that NMDAR antagonism selectively induces aggression in AHA mice, but only up to moderate doses that may be more selective for NMDARs located on parvalbumin interneurons. On the other hand, when the degree of putative NMDAR antagonism crosses a certain threshold, either via extremely high doses of ketamine or memantine, or a combination of alcohol and an antagonist, aggression is inhibited. The researchers went on to show that the effects of systemic memantine are recapitulated by intra-prelimbic PFC infusion of memantine in both AHA and ANA mice, while these effects were not observed after intra-infralimbic PFC memantine infusion. Thus, this final experiment supports the hypothesis that prelimbic PFCspecific NMDAR antagonism is responsible for disinhibiting aggressive behavior. Taken together, research by Newman and colleagues suggests an inverted- $\mathrm{U}$ dose response for prefrontal
NMDAR inhibition-induced aggressive behavior that is strongly shifted to the left in AHA mice, and is likely due to preferential expression of the GluN2D subunit on interneurons. As such only AHA mice exhibit aggression in response to alcohol and selective NMDAR antagonism. In summary, the paper from Newman et al sheds new light on the biological mechanisms of individual vulnerability to alcohol-heightened aggression, and this work is sure to spark new research aimed at reducing the burden of violence on our society.

\section{FUNDING AND DISCLOSURE}

The author declares no conflict of interest.

\section{REFERENCES}

Cherpitel CJ, Ye Y, Bond J, Room R, Borges G (2012). Attribution of alcohol to violence-related injury: self and other's drinking in the event. J Stud Alcohol Drugs 73: 277-284.

van Erp AM, Miczek KA (1997). Increased aggression after ethanol self-administration in male resident rats. Psychopharmacology (Berl) 131: 287-295.

Miczek KA, Barros HM, Sakoda L, Weerts EM (1998). Alcohol and heightened aggression in individual mice. Alcohol Clin Exp Res 22: $1698-1705$.

Morisot N, Ron D (2017). Alcohol-dependent molecular adaptations of the NMDA receptor system. Genes Brain Behav 16: 139-148.

Newman EL, Terunuma M, Wang T, Hewage N, Bicakci MB, Moss $S J$ et al (2018). A role for prefrontal cortical NMDA receptors in murine alcohol-heightened aggression. Neuropsychopharmacology (this issue).

Povysheva NV, Johnson JW (2016). Effects of memantine on the excitation-inhibition balance in prefrontal cortex. Neurobiol Dis 96: $75-83$. 